\title{
High performance InGaAsP lasers fabricated by ion-implantation induced quantum well intermixing
}

\author{
Yen-Ting Pan ${ }^{1 a)}$, San-Liang Lee ${ }^{1}$, Der-Yuh Lin ${ }^{2}$, \\ and Jiun-De $\mathrm{Wu}^{1}$ \\ ${ }^{1}$ Department of Electronic Engineering and Intelligent Building Research Center, \\ National Taiwan University of Science and Technology, Taipei, Taiwan \\ No 43 Keelung $4^{\text {th }}$ Rd., Taipei 10607, Taiwan R.O.C. \\ ${ }^{2}$ Department of Electronic Engineering, National Changhua University of Educa- \\ tion, Changhua, Taiwan
}

No 1, Jin-De Rd., Changhua 50007, Taiwan R.O.C.

a)D9202301@mail.ntust.edu.tw

\begin{abstract}
We demonstrate high-performance InGaAsP based multiple-quantum-well (MQW) lasers fabricated by low-energy ion implantation induced quantum well intermixing (QWI) technique. Different doses of implantation were used to vary the wavelength shift for MQW lasers from the QWI process. At room temperature, the QWI lasers have continuous-wave (CW) characteristics of 10.4-mA threshold current and 13.8-mW maximal output power, which are comparable to the performance of the lasers made of the same as-grown MQW materials. The QWI lasers have a characteristic temperature as high as $58.4 \mathrm{~K}$, which verifies that the material quality after intermixing is feasible for fabricating practical devices.
\end{abstract}

Keywords: high performance, continuous-wave, InGaAsP lasers, lowenergy ion implantation, quantum well intermixing

Classification: Photonics devices, circuits, and systems

\section{References}

[1] S. Charbonneau, E. S. Koteles, P. J. Poole, J. J. He, G. C. Aers, J. Haysom, M. Buchanan, Y. Feng, A. Delage, F. Yang, M. Davies, R. D. Goldberg, P. G. Piva, and I. V. Mitchell, "Photonic integrated circuits fabricated using ion implantation," IEEE J. Sel. Topics Quantum Electron., vol. 4, pp. 772-793, July-Aug. 1998.

[2] E. J. Skogen, J. S. Barton, S. P. Denbaars, and L. A. Coldren, "A quantum-well-intermixing process for wavelength-agile photonic integrated circuits," IEEE J. Sel. Topics Quantum Electron., vol. 8, pp. 863869, July-Aug. 2002.

[3] S. Charbonneau, P. Poole, Y. Feng, G. Aers, M. Dion, M. Davies, R. Goldberg, and I. Mitchell, "Band-gap tuning of InGaAs/InGaAsP/InP laser using high energy ion implantation," Appl. Phys. Lett., vol. 67, pp. 2954-2956, Nov. 1995. 
[4] I. Gontijo, T. Krauss, J. H. Marsh, and R. M. De La Rue, "Postgrowth control of GaAs/AlGaAs quantum well shapes by impurity-free vacancy diffusion," IEEE J. Quantum Electron., vol. 30, pp. 1189-1195, May 1994.

[5] S. Yuan, C. Jagadish, Y. Kim, Y. Chang, H. H. Tan, R. M. Cohen, M. Petravic, L. V. Dao, M. Gal, C. Y. Chan, E. H. Li, J. O, and P. S. Zory, "Anodic-oxide-induced intermixing in GaAs-AlGaAs quantum-well and quantum-wire structures," IEEE J. Sel. Topics Quantum Electron., vol. 4, pp. 629-635, July-Aug. 1998.

[6] T. K. Ong, Y. C. Chan, and B. S. Ooi, "Fabrication of multiple-wavelength lasers in InGaAs-InGaAsP structures using direct laser writing," IEEE Photon. Technol. Lett., vol. 13, pp. 1161-1163, Nov. 2001.

[7] T. K. Sudoh, M. Kumano, Y. Nakano, and K. Tada, "Wavelength trimming by photoabsorption-included disordering for multiple-wavelength distributed-feedback laser arrays," IEEE Photon. Technol. Lett., vol. 9, pp. 887-888, July 1997.

[8] H. S. Lim, V. Aimez, B. S. Ooi, J. Beauvais, and J. Beerens, "A novel fabrication technique for multiple-wavelength photonic-integrated devices in InGaAs- InGaAsP laser heterostructures," IEEE Photon. Technol. Lett., vol. 14, pp. 594-596, May 2002.

[9] M. Paquette, V. Aimez, J. Beauvais, J. Beerens, P. J. Poole, S. Charbonneau, and A. P. Roth, "Blueshifting of InGaAsP-InP laser diodes using a low-energy ion-implantation technique: comparison between strain and lattice-matched quantum-well structures," IEEE J. Sel. Topics Quantum Electron., vol. 4, pp. 741-745, July-Aug. 1998.

[10] J. P. Noël, D. Melville, T. Jones, F. R. Shepherd, C. J. Miner, N. Puetz, K. Fox, P. J. Poole, Y. Feng, E. S. Koteles, S. Charbonneau, R. D. Goldberg, and I. V. Mitchell, "High-reliability blue-shifted InGaAsP/InP lasers," Appl. Phys. Lett., vol. 69, pp. 3516-3518, Dec. 1996.

[11] A. B. Massara, K. A. Williams, I. H. White, R. V. Penty, A. Galbraith, P. Crump, and P. Harper, "Ridge waveguide InGaAsP lasers with uncooled 10 Gbit/s operation at $70^{\circ}$ C," Electron. Lett., vol. 35, pp. 1646-1647, Sept. 1999.

[12] C. W. Hu, F. M. Lee, T. C. Peng, T. M. Ou, M. C. Wu, and Y. H. Huang, "High-Speed and Uncooled Operation of 1.3- $\mu \mathrm{m}$ InGaAsP StrainCompensated MQW BH Lasers Fabricated on Patterned InP Substrates," J. Lightw. Technol., vol. 24, pp. 2906-2911, July 2006.

\section{Introduction}

Quantum well intermixing (QWI) can provide selective-area modification of the heterostructure bandgap on a post-growth wafer. The interdiffusion between the quantum well (QW) and adjacent barrier materials is used for selective control of energy bandgap and other optical properties for the same epitaxial layer structure $[1,2]$. This technique provides the versatility for controlling the bandgap blue-shift by adjusting the implantation temperature, dose, annealing temperature, and annealing time [3].

There have been many reports on various QWI processes, for example, references $[4,5,6]$ and the references therein. For practical device applications, this kind of schemes were successfully demonstrated for realizing the passive sections for sampled grating based tunable lasers [2]. However, rare 
successful laser results were reported when the QWI process was applied on the material of laser gain sections due to the resultant defects from the process $[7,8,9,10]$. In this paper, we apply the implantation-induced QWI process to realize lasers with a large wavelength shift from the gain peak of the as-grown material. Laser sources with high output power, low threshold current, and good characteristic temperature are demonstrated.

\section{Device structures and process conditions}

The QW structure used in this work is an InGaAsP based multiple-quantumwell (MQW) structure of which the as-grown photoluminescence (PL) peak wavelength is about $1573.4 \mathrm{~nm}$ at room temperature. The structure is grown by metal-organic vapor phase epitaxy (MOVPE) on a S-doped InP substrate. The QW laser structure consists of 7 pairs of $1.05 \%$ compressively strained InGaAsP wells and barrier layers in a separate confinement heterostructure (SCH) structure. The QW structure is designed for realizing lasers that can operate at high temperature. The base structure also includes a 100nm thick InP layer, a 55-nm thick InGaAaP stop etch layer, and 470-nm thick InP buffer layer. The buffer layer is designed to completely capture the implanted ions and will be removed after the intermixing process.

The QWI technique includes two steps: ion implantation and thermal annealing. After epitaxial growth of the base structure, phosphorus ions are implanted into the wafers with $100-\mathrm{keV}$ energy. The ion beam is tilted by $7^{\circ}$ with respect to the substrate orientation. Following the implantation, a rapid thermal annealing (RTA) process is carried out on the QWI samples. During annealing, the mobile vacancies induced from implantation can diffuse into the quantum wells and cause intermixing. The annealing was carried out by covering the wafer surface and edges with InP samples to prevent phosphors desorption.

The extent of intermixing depends on both the implantation and annealing process. For realizing high-performance laser diodes with the QWI technique, we choose to use relatively low implantation doses of $1 \times 10^{14} \mathrm{~cm}^{-2}$ and $5 \times 10^{13} \mathrm{~cm}^{-2}$. The implantation was performed as the substrate temperature is elevated to $200^{\circ} \mathrm{C}$. The results of RTA tests reveal that good quality of materials can be retained when the annealing temperature is between $660^{\circ} \mathrm{C}$ to $680^{\circ} \mathrm{C}$. We fixed the annealing temperature at $670^{\circ} \mathrm{C}$ and optimized the annealing time periods.

Fig. 1 (a) show the normalized PL spectra as a function of various annealing time for different implanted doses when the annealing temperature fixed at $670^{\circ} \mathrm{C}$. The relative PL shifts are calculated from the difference between the emission energy of the implanted sample and that of the as-grown reference sample. Fig. 1 (b) shows the variation of PL peak wavelength and the full-width-half-maximum (FWHM) width against the annealing time. It is clear that the wavelength shift in the PL peak is larger for implantation with a larger dose, which creates a greater number of vacancies and allows the exchange of more atoms between QWs and barriers. The wavelength shift for 
the PL peaks can achieve $79.7 \mathrm{~nm}$ for 3 minutes of annealing at $670^{\circ} \mathrm{C}$ when implanted with $1 \times 10^{14} \mathrm{~cm}^{-2}$ dose. The wavelength shift increases with the annealing time. The broadening in the FWHM is minimal, $11.23 \mathrm{meV}$, for 2 minutes of annealing with $5 \times 10^{13} \mathrm{~cm}^{-2}$ dose.

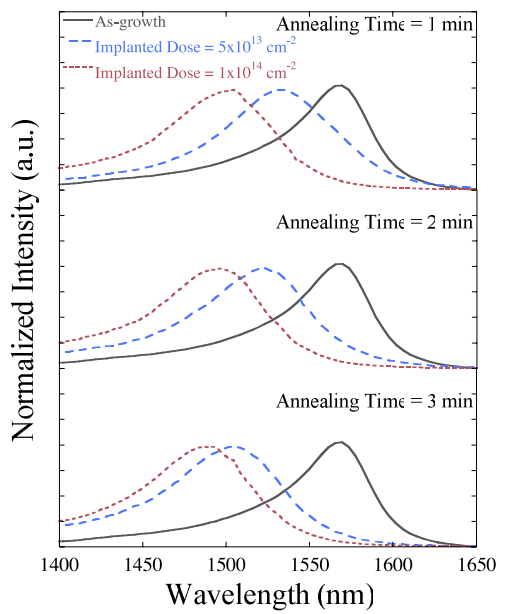

(a)

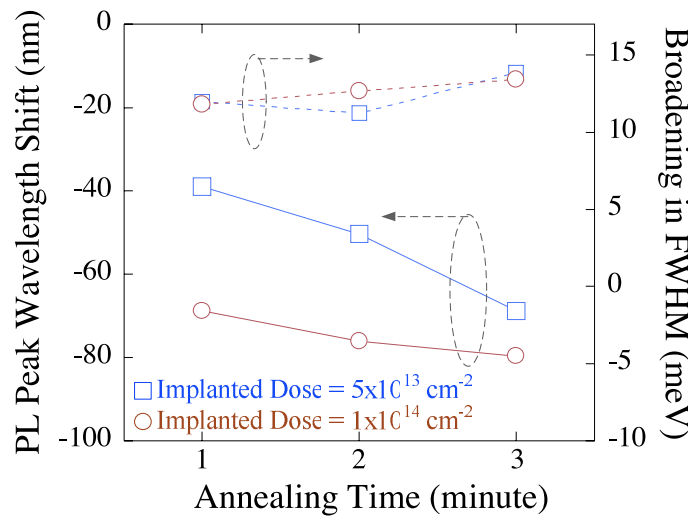

(b)

Fig. 1. (a) Normalized PL spectra (b) variation of the PL peak wavelength and FWHM against the annealing time with the implantation dose of at $5 \times 10^{13} \mathrm{~cm}^{-2}$ and $1 \times 10^{14} \mathrm{~cm}^{-2}$.

\section{Laser fabrication and results}

The QWI lasers are fabricated from wafers implanted at $200^{\circ} \mathrm{C}$ with $5 \times$ $10^{13} \mathrm{~cm}^{-2}$ and $1 \times 10^{14} \mathrm{~cm}^{-2}$ dose, respectively, and annealed at $670^{\circ} \mathrm{C}$ for 2 minutes. After the QWI process, the buffer layer is removed by using wet chemical etching. The cladding layer $(1.55-\mu \mathrm{m}$ thick) and contact layer (0.2$\mu \mathrm{m}$ thick) are then re-grown using MOVPE. The lasers are fabricated into $2 \mu \mathrm{m}$ wide ridge-waveguide Fabry-Perot (FP) lasers. The lasers are cleaved into $300 \mu \mathrm{m}$ long devices and tested under continuous-wave operation.

The light output-current (LI) characteristics of the QWI lasers with cleaved facets at various temperatures are shown in Fig. 2 (a) and Fig. 2 (b) for ion doses of $5 \times 10^{13} \mathrm{~cm}^{-2}$ and $1 \times 10^{14} \mathrm{~cm}^{-2}$, respectively. Lasers for both implantation doses have about the same threshold current $(11.1 \mathrm{~mA}$ and $10.4 \mathrm{~mA}$ ) at $20^{\circ} \mathrm{C}$ while the slope efficiencies is 0.19 and $0.23 \mathrm{~W} / \mathrm{A}$, respectively. Up to $13.8 \mathrm{~mW}$ power can be obtained for the $1 \times 10^{14} \mathrm{~cm}^{-2}$ case. The roll-over in the LI curves is caused by the thermal heating due to poor contact between the bare chips and heat sink. Packaging the lasers can further improve the LI characteristics. The device characteristics prove that good MQW material quality can be maintained after the QWI process.

The comparisons of LI and current-voltage (IV) characteristics among the as-grown lasers and the QWI lasers are shown in Fig. 2 (c). The impedance of the QWI lasers $(14.05 \Omega)$ is slightly larger than that of the as-grown laser 


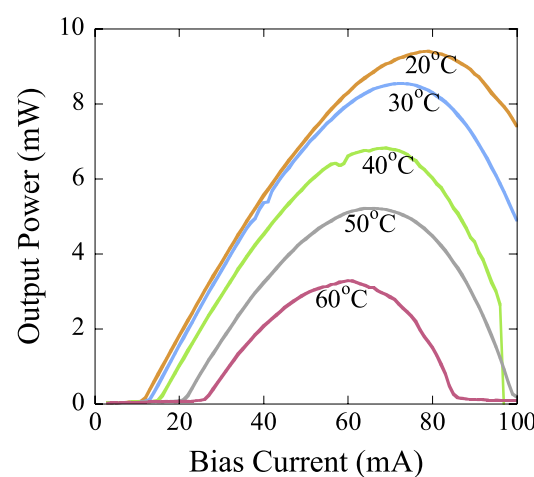

(a)

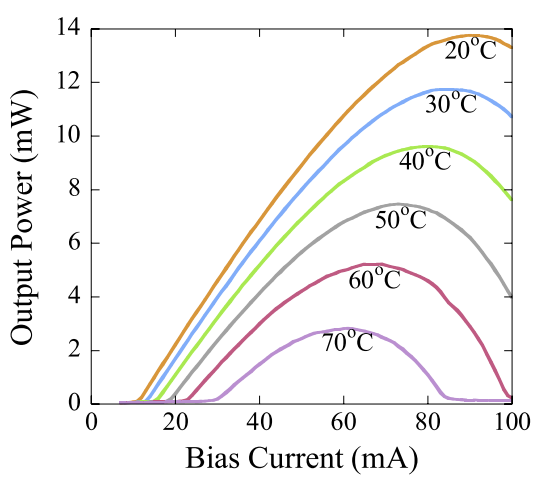

(b)

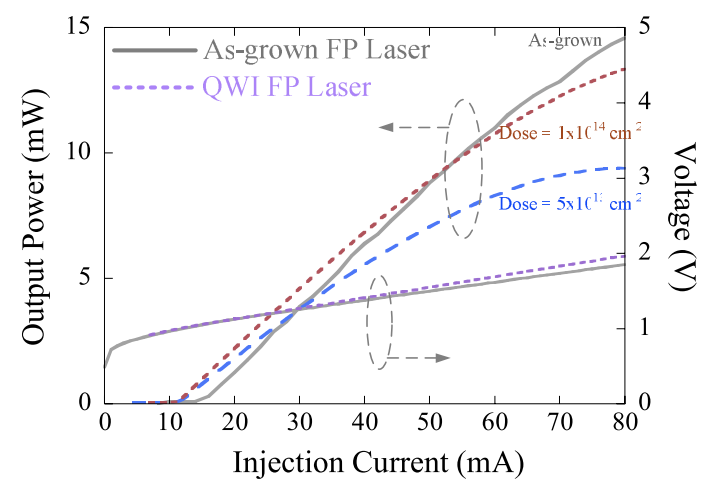

(c)

Fig. 2. CW characteristics for the LD with implanted dose of (a) $5 \times 10^{13} \mathrm{~cm}^{-2}$ (b) $1 \times 10^{14} \mathrm{~cm}^{-2}$ at different operation temperature, and (c) comparison of performance at room temperature with an as-grown laser.

$(12.28 \Omega)$. However, it can be observed from the results that the threshold current of QWI lasers can be as good as the as-grown laser. This again proves good material quality after the QWI process.

Fig. 3 (a) shows the lasing spectrum of as-grown FP laser and QWI FP lasers. The blue shift of peak wavelength is $12.7 \mathrm{~nm}$ and $44 \mathrm{~nm}$ for QWI lasers with an implanted dose of $5 \times 10^{13}$ and $1 \times 10^{14} \mathrm{~cm}^{-2}$, respectively. The difference in the electroluminescence (EL) wavelengths between lasers of the two implanted doses is close to the difference in the PL peak wavelengths. However, the EL wavelength shift from the as-grown laser is less than the PL wavelength shift shown in Fig. 1 (b). This is due to the induced shift in the transition energy by the effect of the applied forward bias. This effect will be addressed elsewhere.

The as-grown laser has a characteristic temperature $\left(\mathrm{T}_{0}\right)$ of around $60 \mathrm{~K}$. Fig. 3 (b) shows the variation of threshold current and slope efficiency with the temperature for the QWI lasers. The characteristic temperature for the implantation dose of $5 \times 10^{13} \mathrm{~cm}^{-2}$ and $1 \times 10^{14} \mathrm{~cm}^{-2}$ is $45.5 \mathrm{~K}$ and $58.4 \mathrm{~K}$, respectively. The results are again close to the typical values of as-grown lasers. To our best knowledge, the results are the first report of QWI lasers 
operating at $70^{\circ} \mathrm{C}$ under $\mathrm{CW}$ condition. This suggests that the intermixing does not severely affect the high-temperature laser characteristics.

The most important consequence of this work is that the QWI lasers can have about the same $\mathrm{T}_{0}$ as the as-grown lasers. Also, the characteristic temperature obtained from the QWI lasers is very promising. InGaAsP based lasers are known to have relative low $\mathrm{T}_{0}$ than AlInGaAs based lasers. The state-of-art InGaAsP based uncooled FP lasers can have $\mathrm{T}_{0}$ of around $70 \mathrm{~K}[11,12]$, which were mostly realized using the buried-heterostructure (BH) waveguide. For comparisons, the QWI lasers are made into simple ridge-waveguide lasers, which typically have larger threshold current than the BH lasers. The QWI lasers of $5 \times 10^{13} \mathrm{~cm}^{-2}$ dose has a slightly lower $\mathrm{T}_{0}$. No obvious difference in the calculated gain characteristics is observed for the gain structures of the two doses by simply considering the parabolic quantum well profiles after the QWI process. The mechanisms that lead to these performance differences require further investigation.

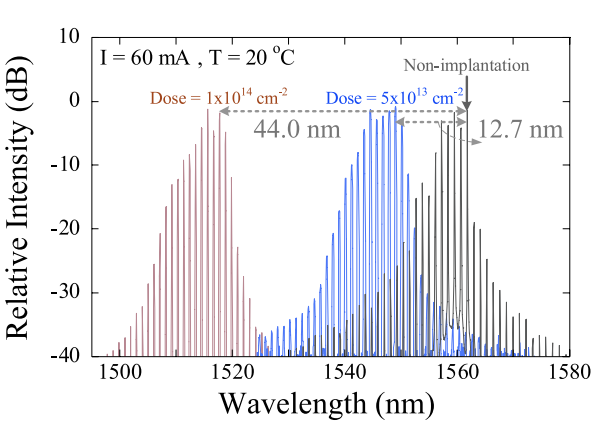

(a)

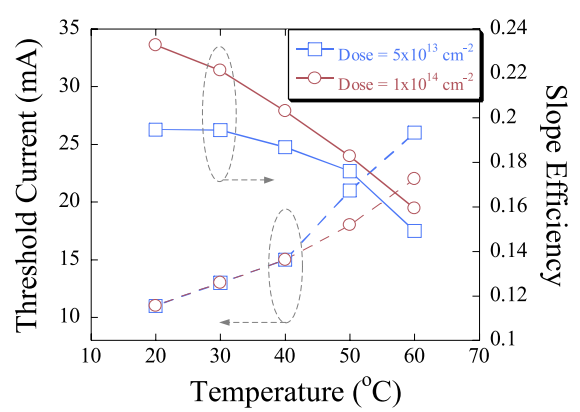

(b)

Fig. 3. (a) Optical spectrum of as-grown FP laser and QWI FP laser and (b) variation of threshold current and slope efficiency against temperature.

\section{Conclusions}

We successfully fabricated high-performance laser diodes in InGaAsP based material systems by using the QWI process that requires low-energy and lowdose ion implantation at elevated substrate temperature. The rapid thermal annealing requires relatively low temperature and relatively short annealing time. More than $79 \mathrm{~nm}$ of wavelength shift on the PL peak is achieved when the implanted dose is $1 \times 10^{14} \mathrm{~cm}^{-2}$. The laser characteristics of low threshold current $(10.4 \mathrm{~mA})$ and high output power $(13.8 \mathrm{~mW})$ under continuous-wave operation indicate good material quality after the QWI process. The characteristic temperature is very close to the typical values of as-grown lasers $(60 \mathrm{~K})$.

The QWI scheme can be applied to realizing lasers of different wavelengths on the same wafer if the selective-area implantation technique is used to vary the implantation dose across the wafer. From the results shown in Fig. 3(a), the two doses together with the as-grown material allow to 
fabricate three DFB lasers with 20-nm wavelength spacing. They can also provide at least $60 \mathrm{~nm}$ of bandgap wavelength range for integrating multiple active/passive components together. More efforts to optimize the operation conditions are still needed in order to improve the versatility of this scheme.

\section{Acknowledgments}

This work was supported in part by National Science Council, Taiwan, R. O. C. under Grant No. NSC 95-2219-E-011-004 and by the Top University Program, Ministry of Education, Taiwan. 